\title{
Erratum
}

\section{AGB stars as tracers of metallicity and mean age across M 33}

\author{
M.-R. L. Cioni ${ }^{1,2}$, M. $\operatorname{Irwin}^{3}$, A. M. N. Ferguson ${ }^{1}$, A. McConnachie ${ }^{4}$, B. C. Conn ${ }^{5}$,

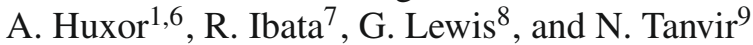 \\ 1 SUPA, School of Physics, University of Edinburgh, IfA, Blackford Hill, Edinburgh EH9 3HJ, UK \\ 2 Centre for Astrophysics Research, University of Hertfordshire, Hatfield AL10 9AB, UK \\ 3 Institute of Astronomy, University of Cambridge, Madingley Road, Cambridge CB3 0HA, UK \\ 4 Deptartment of Physics \& Astronomy, University of Victoria, PO Box 3055, STN CSC, Victoria, BC, V8W 3P6 Canada \\ 5 European Southern Observatory, Alonso de Cordova 3107, Vitacura, Santiago, Chile \\ ${ }^{6}$ Department of Physics, University of Bristol, Tyndall Avenue, Bristol BS8 1TL, UK \\ 7 Observatoire de Strasbourg, 11 rue de l'Université, 67000 Strasbourg, France \\ 8 Institute of Astronomy, School of Physics, A29, University of Sydney, NSW 2006, Australia \\ 9 Department of Physics and Astronomy, University of Leicester, Leicester LE1 7RH, UK
}

A\&A 487, 131-146 (2008), DOI: 10.1051/0004-6361:200809366

Key words. galaxies: individual: M 33 - stars: late-type - galaxies: stellar content - galaxy: abundances galaxies: structure - errata, addenda

The surface distribution of the C/M ratio across M33 is shown in Fig. 1, while Fig. 2 shows the map corresponding to twice a lower resolution. These figures substitute Figs. 10 and 11 in the published version of the paper that show, instead, the distribution of the $\mathrm{M} / \mathrm{C}$ ratio across the galaxy. The new figures show more clearly that regions with higher $\mathrm{C} / \mathrm{M}$ ratio and lower $[\mathrm{Fe} / \mathrm{H}]$ abundance occupy the outer part of the galaxy and delineate a metal-poor ring.

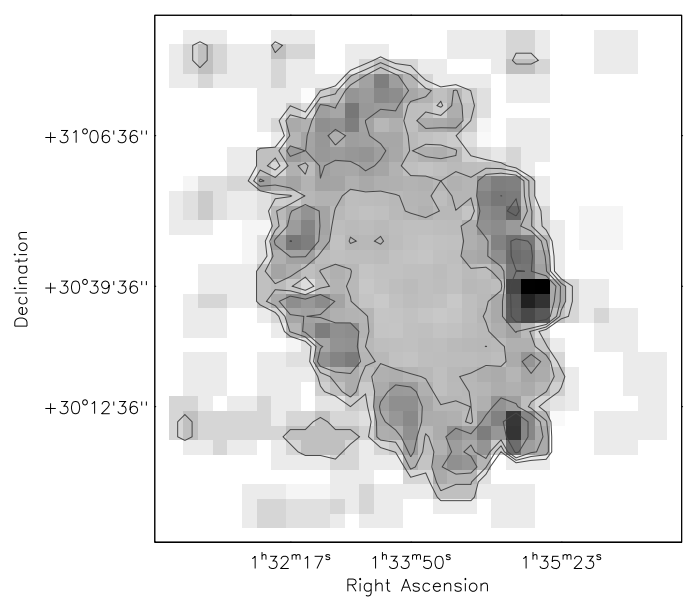

Fig. 2. The same as the bottom panel of Fig. 1 but using bins of 2.4'. Contours are at: $0.28,0.38,0.48$, and 0.68 .
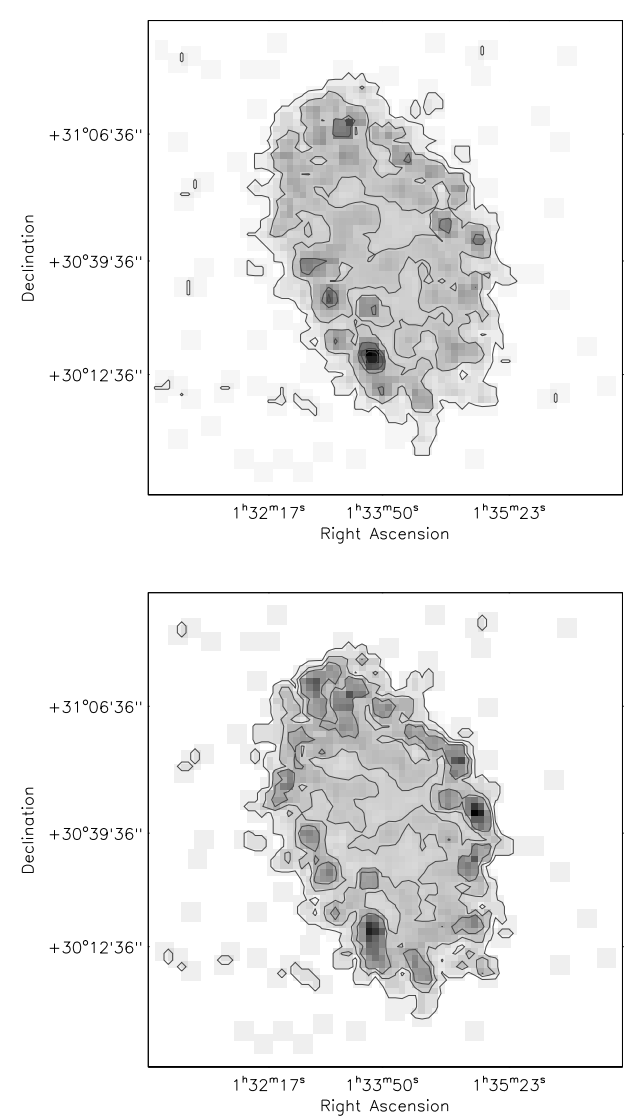

Fig. 1. Distribution of the C/M ratio across M33. C-rich and O-rich AGB stars above the tip of the RGB have been selected using slanted lines (top) or vertical lines (bottom). Darker regions correspond to higher ratios. Contours are at: 0.2, 0.6, 1.2, 1.6, 2.0, and 2.4 in the top panel, and at $0.15,0.35$ and 0.55 in the bottom panel. 